\title{
Multicellular sprouting during vasculogenesis
}

\author{
Andras Czirok, Evan A. Zamir, Andras Szabo, and Charles D. Little \\ Dept. of Anatomy and Cell Biology, University of Kansas Medical Center. Dept. of Biological \\ Physics, Eotvos University
}

\section{Abstract}

Living organisms, from bacteria to vertebrates, are well known to generate sophisticated multicellular patterns. Numerous recent interdisciplinary studies have focused on the formation and regulation of these structures. Advances in automatized microscopy allow the time-resolved tracking of embryonic development at cellular resolution over an extended area covering most of the embryo. The resulting images yield simultaneous information on the motion of multiple tissue components - both cells and extracellular matrix (ECM) fibers. Recent studies on ECM displacements in bird embryos resulted in a method to distinguish tissue deformation and cellautonomous motion.

Patterning of the primary vascular plexus results from a collective action of primordial endothelial cells. The emerging "polygonal" vascular structure is shown to be formed by cell-cell and cellECM interactions: adhesion and protrusive activity (sprouting). Utilizing avb3 integrins, multicellular sprouts invade rapidly into avascular areas. Sprout elongation, in turn, depends on a continuous supply of endothelial cells. Endothelial cells migrate along the sprout, towards its tip, in a vascular endothelial (VE) cadherin-dependent process.

The observed abundance of multicellular sprout formation in various in vitro and in vivo systems can be explained by a general mechanism based on preferential attraction to elongated structures. Our interacting particle model exhibits robust sprouting dynamics and results in patterns with morphometry similar to native primordial vascular plexuses - without ancillary assumptions involving chemotaxis or mechano-chemical signaling.

\section{Introduction}

\section{I.1 Vasculogenesis}

It is widely assumed that adhesion-based activities such as exertion of traction and compressional forces, shape-change and motility are the physical means by which tissues and organs are formed (Trinkaus, 1984; Forgacs and Newman, 2005). However, our knowledge is limited about how collective cell behavior creates a specific physical tissue or organ (Keller et al., 2003).

Vasculogenesis of warm blooded vertebrates, the formation of a primary vascular pattern from mesodermal-derived precursors (angioblasts), is an excellent system in which to address tissue pattern emergence. In bird embryos, well before the onset of circulation, hundreds of essentially identical vascular endothelial cells create a polygonal network within a relatively uncomplicated, sheet-like anatomical environment (Risau and Flamme, 1995). Each link in the polygonal network is a cord consisting of 3-10 endothelial cells (Drake et al., 1997).

It has been known for nearly a century that endothelial cells differentiate from solitary angioblasts within the avian embryo (area pellucida) (Reagan, 1915; Sabin, 1920). Committed angioblasts display a random spatial distribution within the mesoderm, without 
an observable preexisting pattern at length scales comparable to that of the future primary vascular polygons (Drake et al., 1997).

Vasculogenic processes may also occur in certain pathophysiologies. Recent data provided evidence that vascular endothelial cell progenitors exist in the adult and may become bloodborne, enter extravascular tissues, and promote de novo vessel formation (Zammaretti and Zisch, 2005; Rumpold et al., 2004). For that reason, endothelial progenitors, mobilized in situ or transplanted, are a major target of therapeutic vascularization approaches to prevent ischemic disease and control endothelial injury. Moreover, endothelial progenitors represent a potential target for strategies to block tumor growth, and a requisite mechanism for tissue engineering (Wu et al., 2004). The capacity of less than fully differentiated cells to assemble into vascular-like tubes is also manifested in various tumors. Best characterized are highly malignant melanomas in which tumor cells are assembling into tubes to secure blood supply (Hendrix et al., 2003). Thus, arguably, the least understood and most important question facing vascular developmental biologists and tissue engineers today is - what are the general principles guiding morphogenesis of an endothelial tube network?

\section{I.2 Self-organization vs genetic hard-wiring}

Conventional models of vasculogenesis often assume that endothelial cells migrate to preand well-defined positions following extracellular guidance cues or chemoattractants (Ambler et al., 2001; Cleaver and Krieg, 1998; Poole and Coffin, 1989). However, the capacity of endothelial cells to form a polygonal pattern is preserved in various in vitro systems, where the presence of a genetic pre-pattern is unlikely. The mouse allantois, when explanted, forms a vascular network very similar to the primary vascular pattern (LaRue et al., 2003) - instead of a pair of umbilical vessels. Similarly, a polygonal vascular network emerges when endothelial cells are placed in three dimensional collagen gels (Montesano and Orci, 1985; Davis et al., 2000). We argue that the ubiquitous polygonal networks are self-organized in the sense that we do not expect a direct correspondence between gene expression patterns and the position of individual segments within the primary vascular network.

This concept of self-organization is markedly different from that of genetic hard-wiring. During insect segmentation the position and identity of body segments is directly correlated with a gene expression pattern. Similarly, genetic pre-patterning is clearly involved in the determination of major vessels in the developing vascular network. In fish, where the major vessels assemble directly, without forming an intermediate vascular plexus, specific vascular malformations are correlated with genetic defects (Weinstein, 1999). Thus, endothelial progenitors presumably respond to various environmental cues, including the presence of other endothelial cells as well as genetically pre-programmed "zip codes".

\section{I.3 Theories of self-assembly}

Various hypotheses are proposed to explain the self-organized aspect of vasculogenesis. The mechano-chemical mechanism assumes cells to exert mechanical stress on the underlying substrate, and the resulting stress to guide cell motility (Murray et al., 1998; Murray, 2003). A variant of the mechano-chemical model proposes that angioblasts first segregate into compact clusters and engage the surrounding ECM fibers. As a result of traction forces, ECM bundles develop, which in turn later route the motile primordial endothelial cells between clusters (Drake et al., 1997; Manoussaki et al., 1996; Vernon et al., 1995).

A recent body of research focused on pattern emergence based on autocrine chemotactic signaling (Gamba et al., 2003; Serini et al., 2003). The suggested chemoattractant, $\mathrm{VEGF}_{165}$, is unlikely to fit the model assumptions in embryonic vasculogenesis as $\mathrm{VEGF}_{165}$ 
is expressed throughout the embryo except in endothelial cells (Flamme et al., 1995; Poole et al., 2001). However, patterning guided by an unspecified chemoattractant continues to serve as the basis of biologically plausible models resolving individual cells (Merks et al., 2006) as well as freely diffusive or matrix-bound chemoattractants (Bauer et al., 2007).

Recently we proposed that mechanical effects may also modulate cell migration guided by adjacent cells, a view we will further explore in chapter III.

\section{Empirical data, in vivo}

Most of the experimental biology underpinning the mathematical models has relied on in vitro studies. However, vascular morphogenesis occurs at times and places in avian embryos that are readily observed and manipulated in vivo (Little and Drake, 2000). Due to recent improvements in digital microscopy, it is now possible to address directly how new blood vessels form de novo (Czirok et al., 2002; Rupp et al., 2003a). Scanning time-lapse microscopy allows both the global (tissue-scale) and local (cell-autonomous) characterization of primordial endothelial cell behavior during the initial stages of avian vasculogenesis.

Endothelial cells are tracked by use of microinjected and fluorochrome-conjugated QH1 antibodies, specific for quail vascular endothelium (Pardanaud et al., 1987). At each time point images can be taken in multiple optical modes: one for QH1 fluorescence, another visualizing the extracellular matrix (ECM) environment by using an immunolabelled ECM component. A third image, bright field or differential interference contrast (DIC), is also acquired to provide an anatomical frame of reference. The resulting images are aligned such that certain anatomical reference points, e.g. the intersomitic clefts remain stationary. Furthermore, due to the availability of images from multiple focal planes, no object is lost or rendered out of focus.

\section{II.1 Vascular drift}

Early vasculogenesis is a time of vigorous rearrangements in the embryo, with parallel processes occurring at various length scales from the migration of individual cells to global morphogenic events, such as gastrulation or neurulation. These tissue movements, which can be mapped using ECM fibrils as passive tracers (Zamir et al., 2006), profoundly influence vascular pattern formation. As Rupp et al. (2004) and Fig. 1 demonstrates, there is a substantial medial movement of the forming vascular plexus, a phenomenon predicted by Coffin and Poole (1988). This process, vascular drift, was shown to occur within almost the entire nascent vasculature. A comparison of changes in JB3 anti-fibrillin 2 (Rongish et al., 1998) and endothelium-specific QH1 immuno-fluorescence reveals that the motion of vascular segments is largely coincident with the global tissue flow accompanying gastrulation and elongation of the notochord (Fig. 1). Therefore, vascular structures appear as embedded in a mechanical continuum.

Morphogenetic flows in the embryo have a profound impact on tissue structure. One experimentally accessible measure of tissue structure is its two-dimensional (2D) ECM- or cell density. This measure gives the number of objects, projected onto a frontal plane, per unit area. As we demonstrated (Czirok et al., 2006), morphogenetic movements accumulate tissue components around the somites. This increase in 2D density can reflect both an accumulation of cells and ECM, and a general expansion in tissue thickness. As Fig. 2 demonstrate, large-scale tissue movements indeed accumulate endothelial cells. As there is a strong correlation between 2D cell density and network morphology (LaRue et al., 2003), changes in endothelial cell density are associated with structural rearrangements of the vascular network. The most frequently observed manifestation of a structural rearrangement 
is vascular fusion (Drake and Little, 1999), whereby distinct polygons fuse to form a common lumen, as in the case of large vessels such as the sinus venosus, or the dorsal aortae (see Fig. 1 of Rupp et al. (2004)). Tissue displacements also alter network morphology by imposing substantial strain along the embryonic axis aligning the segments of the nascent plexus (Fig. 2).

\section{II.2 Vasculogenic sprouts}

Endothelial cells are also capable of active motion, relative to their ECM surroundings. The early vascular plexus is characterized by disconnected endothelial clusters. To establish the network, endothelial cells send out extensions across the avascular ECM. As demonstrated in Fig. 1, the protrusions (vasculogenic sprouts) typically move medially, and much faster than the drift of the ECM. This type of protrusive behavior is reminiscent of angiogenic sprouting, a process thought to be characteristic of later vascular development.

As analyzed in detail by Rupp et al. (2004), vasculogenic sprouts can contact neighboring extensions and thus establish endothelial cords. A stabilized protrusion may later be reinforced by subsequent addition of cells - making thereby new vertices and segments in the primary vascular pattern. As sprouts can extend hundreds of micrometers (Fig. 3), they are presumably multicellular structures and thus sprout extension involves the coordinated activity of several (3-10) endothelial cells. Conversely, existing connections are also observed to retract, albeit with much less frequency.

The empirical motility data thus reveal that protrusive activity or sprouting is the mechanism used to generate new vascular cords resulting in the polygonal vascular pattern. Only a small fraction of endothelial cells exhibit this type of protrusive behavior, and within a welldefined developmental stage (Rupp et al., 2004). Furthermore, simultaneous monitoring of endothelial sprouts and changes in the surrounding ECM configuration revealed no evidence for sprout guidance by local ECM deformations (Fig. 1) - in contrast with the predictions of the mechano-chemical models.

\section{II.3 Cell-cell adhesion}

By subtracting the overall medial drift of the vascular pattern, trajectories of individual endothelial cells, relative to the surrounding endothelial structures, can be established (Fig. 4). Each labeled cell can be traced back either to a cluster of endothelial cells or to an avascular area, where they appear de novo. These newly recruited cells move quickly until incorporation into an existing vascular structure occurs. Once part of a vascular cord, their speed is usually reduced, and when moving, they remain in close vicinity of other endothelial cells. The course of individual motile cells was found to be highly persistent along the vascular segments. There is a significant variation of motile activity within the endothelial cell population, as the substantially differing trajectories demonstrate.

Most endothelial cells move in a medial direction (Rupp et al., 2003b, 2004), which is also preferred by vasculogenic sprouts. This combination of medial migration along vascular segments and medial sprouting increases the apparent speed of vascular drift above that of tissue movements (Rupp et al., 2004). This increase occurs despite the fact that the vascular structure is embedded into, and moves with, the surrounding ECM as Fig 1 demonstrates. The two types of endothelial cell motility (sprout vs. individual) differ mainly with respect to their substrates: while the protrusive activity is integrin dependent and requires an active engagement of the ECM (Rupp et al., 2004), endothelial cell motility along existing vascular structures appears to rely more upon vascular endothelial (VE)-cadherin mediated cell-cell interactions (Perryn et al., in preparation; PhD dissertation, 2006). 


\section{Elongated structures, in vitro}

The formation of linear segments via sprout-like activity, however, is not restricted to vascular endothelial cells (Szabo et al., 2007). As Fig. 5 demonstrates, non-vascular C6 cells, along with muscle- or fibroblast-related cells (data not shown) also exhibit linear structures when grown on a rigid plastic tissue culture substrate in the presence of a continuously shaken culture medium. Depending on the cell density, the linear segments merge and form a network. Compared to the primary vascular pattern, the resulting network appears to be more irregular due to the wider distribution of cell-free areas or segment lengths and widths. Nevertheless, the distribution of cells is far from random, and favors linear configurations.

The non-random placement of cell bodies can be characterized by the following morphometric procedure for local anisotropy. A diffusive process is started from various points of the segmented, and subsequently smoothened (long pass-filtered), image (see the diagram in Fig. 6). The selected point acts as steady point-source of the diffusive field. The diffusion coefficient is zero below a concentration threshold, thus a well-defined front emerges which encloses a gradually increasing area around the point source. The growing area is characterized by the principal moments of its inertia, $\lambda_{1}$ and $\lambda_{2}$, where $\lambda_{1}>\lambda_{2}$. If the area is circular or isotropic, these principal moments are of a similar magnitude. In contrast, highly elongated anisotropic structures result in dissimilar principal moments. The area is grown until its width, $\lambda_{2}^{3 / 8} \lambda_{1}^{-1 / 8}$, reaches a pre-defined value, the typical width of a single cell. The local anisotropy of the location at the point-source is then characterized as $\sqrt{\lambda_{1} / \lambda_{2}}$. Repeating the procedure for various positions results in an anisotropy map, as shown in Fig. $5 c$.

To correlate the average anisotropy index with local cell density, anisotropy maps were obtained from 15 independent microscopic fields of C6 cells. Cell density was characterized by the local volume fraction (area covered by cells on the segmented image, divided by the size of the local area). For convenience, the volume fraction values are rescaled such that a confluent cell layer has a relative volume fraction value of 1 . As Fig 5d reveals, areas with high cell densities have an isotropic structure. Very low cell densities prohibit the formation of multicellular clusters. Between these two extremes, at $20 \%$ confluency (relative volume fraction 0.2), cell arrangements have a significantly higher anisotropy index $(p<0.01)$ than in the corresponding randomized sample where each cell body is randomly placed, without overlaps, within the same area.

\section{Mathematical model of sprout formation}

As shown in section II and by Szabo et al. (2007), linear multicellular structures form via sprouting both during in vivo vascular patterning, and in simple in vitro cell cultures. Patterning through sprouting is markedly different from the gradual coarsening of an initially uniform density field, and its possible arrest, characteristic for colloid gels (see, e.g. (Foffi et al., 2005)) or for several models proposed to describe vasculogenesis. In particular, a "frozen" pattern was reported to gradually emerge, with an increasing spread in cell density, in the mechano-chemical model (see Fig. 6, of Manoussaki et al. (1996), or Fig. 7 of Namy et al. (2004)). Gradually increasing avascular area sizes were reported in the autocrine chemoattractant model (Fig. 1 of Serini et al. (2003)). Except for a recent model with autocrine chemotaxis and contact inhibition (Merks et al., 2007), none of these models were reported to produce sprouts - most likely because they aimed to reproduce a specific in vitro model system where endothelial cells are placed on the surface of a malleable gel (Vernon et al., 1995). 
While both the mechano-chemical and chemoattractant mechanisms may be biologically relevant under certain circumstances, neither mechanism is expected to operate within the simple in vitro experimental setup of Section III. The rigid substrate excludes the mechanochemical mechanism. A specific chemotactic response is unlikely to be shared by such a variety of cell types. Finally, convection currents in the culture medium, generated by temperature inhomogeneities within the incubator and the vibrations of microscope stage motion, are expected to hamper the maintenance of concentration gradients, or impose a strong directional bias upon the chemotaxis-related cell movements (Szabo et al., 2007).

Motivated by the empirical observations on sprout expansion guided by adjacent projections of other cells or elongated multicellular structures, recently we proposed that multicellular sprouting may employ a general mechanism, the preferential attraction to elongated structures. While the molecular basis of such a behavior is unknown, one may conjecture that cells in elongated structures are under mechanical tension, and strained cells can have a stiffer cytoskeleton (Xu et al., 2000). Cells are able to respond to variations in extracellular matrix stiffness (Gray et al., 2003), and an analogous mechanotaxis utilizing cell-cell contacts is also feasible. For example, VE-cadherin, a major cell-cell adhesion receptor of vascular endothelial cells, was recently shown to be incorporated in cell surface mechanosensing complexes (Tzima et al., 2005).

\section{IV.1 Equation of motion}

To assess the collective behavior of cells exhibiting the proposed preferential attraction property, a simple model was studied in which individual cells were represented as particles. Cell motility is often approximated as a persistent, Ornstein-Uhlenbeck diffusion process (Stokes et al., 1991; Selmeczi et al., 2005), where the velocity $\vec{v}_{k}$ of cell $k$ is described by the Langevin equation

$$
\frac{d \vec{v}_{k}}{d t}=-\vec{v}_{k} / \tau+\sqrt{D} \xi_{k}+\vec{M}_{k}
$$

where $\tau$ and $D$ are parameters specifying the persistence time and the randomness of motion, respectively. The variable $\xi$ represents an uncorrelated white noise: $\langle\xi\rangle=0$ and $\left\langle\xi_{k}(t) \xi_{l}\left(t^{\prime}\right)\right\rangle=$ $\delta_{k l} \delta\left(t-t^{\prime}\right)$. Term $\vec{M}$ is a deterministic bias, representing interaction with the environment, that is, with adjacent particles.

While Eq.(1) describes the motion of a Brownian particle at finite temperatures, animal cell motility is driven by complicated molecular machinery, and it is not thermal fluctuation driven. Thus, parameters $\tau$ and $D$ depend substantially on cell type and molecular state. Measurements performed with non-interacting endothelial cells and fibroblasts resulted $\tau$ and $D$ values in the $0.1-5 \mathrm{~h}$ and $100-2000 \mu \mathrm{m}^{2} / \mathrm{h}^{3}$ range, respectively (Dunn, 1983;Stokes et al., 1991).

\section{IV.2 Interaction with the environment}

Interactions among mobile agents are usually modelled as a sum of pair interactions (Helbing, 2001). In this spirit, $\vec{M}$ is factored into

$$
\vec{M}_{k}=\sum_{\{j\}} \frac{\vec{x}_{j}-\vec{x}_{k}}{d_{k j}}\left[f_{1}\left(d_{k j}\right)+w_{j} f_{2}\left(d_{k j}\right)\right],
$$


where the sum is taken over the Voronoi neighbors of particle $k$, and $d_{k j}=\left|\vec{x}_{j}-\vec{x}_{k}\right|$ (see Fig. 7). The repulsion term $f_{1}$ ensures that model cells are impenetrable. The range of repulsion is the size $R_{1}$ of the organelle-packed region around the cell nucleus. The preferential attraction response is incorporated in the $f_{2}$ term. Cells are expected to explore their surroundings with protrusions as proposed by Flamme et al. (1993), and respond when protrusions contact elongated structures. Filopodia typically extend from $R_{2}$, the cell surface $\left(R_{1} \leq R_{2}\right)$, to a maximal distance of $R$. Thus, $f_{1}(d)=0$ for $d>R_{1}$ and $f_{2}(d)=0$ for $d<R_{2}$ and $d>R$. We estimate $R_{1}=10 \mu \mathrm{m}, R_{2}=30 \mu \mathrm{m}$ and $\mathrm{R}=40 \mu \mathrm{m}$. These values, however, can vary by at least a factor of two, depending on the cell types, shapes and experimental conditions.

There is little empirical guidance on the choice of functions $f_{1}$ and $f_{2}$. Among other functions, Szabo et al. (2007) assumed a linear repulsion $f_{1}(d)=-A\left(R_{1}-d\right)$ and a zonal, distance-independent attraction $f_{2}(d)=B$ for $R_{2}<d<R$, where $A$ and $B$ are parameters. The representative simulation results of Figs 8 . and 9 were obtained with parameters $A=160 \mathrm{~h}^{-2}$ and $B=130 \mu \mathrm{m} / \mathrm{h}^{2}$. These values represent a strong response to external cues: the ratio of the directed and random velocity components is $B \tau / \sqrt{D \tau} \approx 3$. As a comparison, a similar measure for chemotactic response of endothelial cells was found slightly larger than one (Stokes et al., 1991).

In the case of generic cell adhesion $\left(w_{k}=1 / 2\right)$ the model was reported to behave as a twodimensional, colloid-like system (Szabo et al., 2007). Most colloid systems with large enough attraction range exhibit transient gels; the pattern coarsens and eventually collapses into globular clusters (Butler et al., 1995). This behavior is indeed exhibited by some cell types, as cell sorting experiments demonstrate (Beysens et al., 2000).

For the sake of simplicity, cell shape is not explicitly resolved in the model. Therefore, elongation or local anisotropy is inferred from the configuration of particles. To represent an attraction to cells within anisotropic structures, the weights $w_{k}$ are constructed as

$$
w_{k}=\frac{1}{n_{k}}\left|\sum_{\left\{j: d_{j k}<R\right\}} e^{2 i \varphi_{j k}}\right|^{2}
$$

where the sum is taken over all $n_{k}$ particles that are within a circle of radius $R$ around particle $k$. The angle between $\vec{x}_{k}-\vec{x}_{j}$ and an arbitrary reference direction is denoted by $\varphi_{j k}$. Thus, $w=0$ for particles in an isotropic environment and $w=1$ for particles in a highly elongated, linear configuration. Non-uniform weights result in asymmetric pair-interactions, which is feasible as $\vec{M}$ represents a bias in cell activity instead of physical forces.

\section{IV.3 Simulation results}

When the initial condition is a single, dense cluster, stable branches form and elongate, reminiscent of the sprout formation observed in vivo or in vitro (Fig. 8). If the particles are randomly positioned at $t=0$, then long, linear arrays form after an initial coarsening process (Fig. 9). At sufficiently high particle densities the linear segments interconnect and form a polygonal network (Fig. 10). After the initial transient the pattern becomes quasi-stationary: the generation of new branches balances the surface-tension driven coarsening and elimination of holes. The characteristic pattern size was found to be insensitive to the cell density (Szabo et al., 2007), in good agreement with the somewhat limited morphometric data available for the vasculature of quail embryos (LaRue et al., 2003). 


\section{Conclusions}

This chapter demonstrates that the primary vascular plexus of warm blooded vertebrates is formed through processes operating on various length scales: including sprouting and tissue movements. The formation and rapid expansion of multicellular sprouts is the key mechanism by which disconnected endothelial cell clusters join to form an interconnected network. As the simulation results demonstrate, the proposed hypothesis of cellular attraction to elongated structures can explain a number of empirically observed features of multicellular sprout formation. Thus, it is likely to complement other mechanisms such as chemotaxis and interaction with the surrounding ECM in the determination of endothelial cell behavior.

\section{Acknowledgments}

We are grateful to Tracey Cheuvront, Mike Filla and Alan Petersen for their technical expertise. This work was supported by the G Harold and Leila Y Mathers Charitable Foundation (to C.D.L.), the NIH (R01 HL68855 to C.D.L., R01 HL87136 to A.C.), the American Heart Association (Scientist Development Grant $0535245 \mathrm{~N}$ to A.C, Heartland Affiliate postdoctoral fellowship to E.A.Z) and the Hungarian Research Fund (OTKA T047055, to A.C.).

\section{References}

Ambler CA, Nowicki JL, Burke AC, Bautch VL. Assembly of trunk and limb blood vessels involves extensive migration and vasculogenesis of somite-derived angioblasts. Dev Biol 2001;234:352-364. [PubMed: 11397005]

Bauer AL, Jackson TL, Jiang Y. A cell-based model exhibiting branching and anastomosis during tumor-induced angiogenesis. Biophys J. 2007

Beysens DA, Forgacs G, Glazier JA. Cell sorting is analogous to phase ordering in fluids. PNAS 2000;97:9467-9471. [PubMed: 10944216]

Butler B, Hanley H, Hansen D, Evans D. Dynamic scaling in an aggregating 2d lennardjones system. Phys. Rev. Lett 1995;74:4468-4471. [PubMed: 10058514]

Cleaver O, Krieg PA. Vegf mediates angioblast migration during development of the dorsal aorta in xenopus. Development 1998;125:3905-3914. [PubMed: 9729498]

Coffin D, Poole T. Embryonic vascular development: immunohistochemical identification of the origin and subsequent morphogenesis of the major vessel primordia in quail embryos. Development 1988;102:735-748. [PubMed: 3048971]

Czirok A, Rupp P, Rongish B, Little C. Multi-field 3D scanning light microscopy of early embryogenesis. J Microsc 2002;206:209-217. [PubMed: 12067365]

Czirok A, Zamir EA, Filla MB, Little CD, Rongish BJ. Extracellular matrix macroassembly dynamics in early vertebrate embryos. Current Topics in Developmental Biology 2006;73 (in press).

Davis GE, Black SM, Bayless KJ. Capillary morphogenesis during human endothelial cell invasion of three-dimensional collagen matrices. In Vitro Cell Dev Biol Anim 2000;36:513-519. [PubMed: 11149750]

Drake CJ, Brandt SJ, Trusk TC, Little CD. Tal1/scl is expressed in endothelial progenitor cells/ angioblasts and defines a dorsal-to-ventral gradient of vasculogenesis. Dev Biol 1997;192:17-30. [PubMed: 9405094]

Drake CJ, Little CD. Vegf and vascular fusion: implications for normal and pathological vessels. J Histochem Cytochem 1999;47:1351-1356. [PubMed: 10544208]

Dunn GA. Characterising a kinesis response: time averaged measures of cell speed and directional persistence. Agents Actions Suppl 1983;12:14-33. [PubMed: 6573115]

Flamme I, Baranowski A, Risau W. A new model of vasculogenesis and angiogenesis in vitro as compared with vascular growth in the avian area vasculosa. Anat Rec 1993;237:49-57. [PubMed: 7692767] 
Flamme I, Breier G, Risau W. Vascular endothelial growth factor (vegf) and vegf receptor 2 (flk-1) are expressed during vasculogenesis and vascular differentiation in the quail embryo. Dev Biol 1995;169:699-712. [PubMed: 7781909]

Foffi G, De Michele C, Sciortino F, Tartaglia P. Arrested phase separation in a shortranged attractive colloidal system: a numerical study. J Chem. Phys 2005;122 224903.

Forgacs, G.; Newman, SA. Biological Physics of the Developing Embryo. Cambridge: Cambridge University Press; 2005.

Gamba A, Ambrosi D, Coniglio A, de Candia A, Di Talia S, Giraudo E, Serini G, Preziosi L, Bussolino F. Percolation, morphogenesis, and burgers dynamics in blood vessels formation. Phys Rev Lett 2003;90:118101. [PubMed: 12688968]

Gray D, Tien J, Chen C. Repositioning of cells by mechanotaxis on surfaces with micropatterned young's modulus. J Biomed Mater Res A 2003;66:605-614. [PubMed: 12918044]

Hamburger V, Hamilton H. A series of normal stages in the development of the chick embryo. J. Morphol 1951;88:49-92.

Helbing D. Traffic and related self-driven many-particle systems. Rev. Mod. Phys 2001;73:10671141.

Hendrix MJC, Seftor EA, Hess AR, Seftor REB. Vasculogenic mimicry and tumour-cell plasticity: lessons from melanoma. Nat Rev Cancer 2003;3:411-421. [PubMed: 12778131]

Keller R, Davidson LA, Shook DR. How we are shaped: the biomechanics of gastrulation. Differentiation 2003;71:171-205. [PubMed: 12694202]

LaRue AC, Mironov VA, Argraves WS, Czirok A, Fleming PA, Drake CJ. Patterning of embryonic blood vessels. Dev Dyn 2003;228:21-29. [PubMed: 12950076]

Little C, Drake C. Whole-mount immunolabeling of embryos by microinjection. Methods Mol. Biol 2000;135:183-189. [PubMed: 10791315]

Manoussaki D, Lubkin S, Vernon R, Murray J. A mechanical model for the formation of vascular networks in vitro. Acta Biotheor 1996;44(3-4):271-282. [PubMed: 8953213]

Merks R, Perryn E, Glazier J. Contact-inhibited chemotactic motility: Role in de novo and sprouting blood vessel growth. arXiv. 20070505033.

Merks RM, Brodsky SV, Goligorksy MS, Newman SA, Glazier JA. Cell elongation is key to in silico replication of in vitro vasculogenesis and subsequent remodeling. Dev Biol 2006;289:44-54. [PubMed: 16325173]

Montesano R, Orci L. Tumor-promoting phorbol esters induce angiogenesis in vitro. Cell 1985;42:469-477. [PubMed: 2411423]

Murray, JD. Mathematical Biology. 2 edn.. Berlin: Springer Verlag; 2003.

Murray, JD.; Manoussaki, D.; Lubkin, SR.; Vernon, R. A mechanical theory of in vitro vascular network formation. In: Little, CD.; Mironov, V.; Sage, EH., editors. Vascular morphogenesis: In vivo, in vitro, in mente. Boston: Birkhauser; 1998. p. 223-239.

Namy P, Ohayon J, Tracqui P. Critical conditions for pattern formation and in vitro tubulogenesis driven by cellular traction fields. J Theor Biol 2004;227:103-120. [PubMed: 14969709]

Pardanaud L, Altmann C, Kitos P, Dieterlen-Lievre F, Buck C. Vasculogenesis in the early quail blastodisc as studied with a monoclonal antibody recognizing endothelial cells. Development 1987;100:339-349. [PubMed: 3308401]

Poole T, Coffin J. Vasculogenesis and angiogenesis: Two distinct morphogenetic mechanisms establish embryonic vascular pattern. J. Exp. Zool 1989;251:224-231. [PubMed: 2671254]

Poole TJ, Finkelstein EB, Cox CM. The role of fgf and vegf in angioblast induction and migration during vascular development. Dev Dyn 2001;220:1-17. [PubMed: 11146503]

Reagan F. Vascularization phenomena in fragments of embryodic bodies completely isolated from yolk sac blastoderm. Anat. Rec 1915;9 329-241.

Risau W, Flamme I. Vasculogenesis. Annu. Rev. Cell Dev. Biol 1995;11:73-91. [PubMed: 8689573]

Rongish B, Drake C, Argraves W, Little C. Identification of the developmental marker, JB3-antigen, as fibrillin-2 and its de novo organization into embryonic microfibrous arrays. Dev. Dyn 1998;212:461-471. [PubMed: 9671949] 
Rumpold H, Wolf D, Koeck R, Gunsilius E. Endothelial progenitor cells: a source for therapeutic vasculogenesis? J Cell Mol Med 2004:509-518. [PubMed: 15601579]

Rupp P, Rongish B, Czirok A, Little C. Culturing of avian embryos for time-lapse imaging. Biotechniques 2003a;34:274-278. [PubMed: 12613250]

Rupp PA, Czirok A, Little CD. Novel approaches for the study of vascular assembly and morphogenesis in avian embryos. Trends Cardiovasc Med 2003b;13:283-288. [PubMed: 14522468]

Rupp PA, Czirok A, Little CD. alphavbeta3 integrin-dependent endothelial cell dynamics in vivo. Development 2004;131:2887-2897. [PubMed: 15151986]

Sabin F. Studies on the origin of the blood vessels and of red blood corpusles as seen in the living blastoderm of chick during the second day of incubation. Carnegie Contrib. Embryol 1920;9:215262.

Selmeczi D, Mosler S, Hagedorn PH, Larsen NB, Flyvbjerg H. Cell motility as persistent random motion: theories from experiments. Biophys J 2005;89:912-931. [PubMed: 15951372]

Serini G, Ambrosi D, Giraudo E, Gamba A, Preziosi L, Bussolino F. Modeling the early stages of vascular network assembly. EMBO J 2003;22:1771-1779. [PubMed: 12682010]

Stokes CL, Lauffenburger DA, Williams SK. Migration of individual microvessel endothelial cells: stochastic model and parameter measurement. J Cell Sci 1991;99:419-430. [PubMed: 1885678]

Szabo A, Perryn ED, Czirok A. Network formation of tissue cells via preferential attraction to elongated structures. Phys Rev Lett 2007;98 038102.

Trinkaus, J. Cells into Organs. The forces that shape the embryo. 2nd edn. Englewood Cliffs, New Jersey: Prentice-Hall, Inc.; 1984.

Tzima E, Irani-Tehrani M, Kiosses WB, Dejana E, Schultz DA, Engelhardt B, Cao G, DeLisser H, Schwartz MA. A mechanosensory complex that mediates the endothelial cell response to fluid shear stress. Nature 2005;437:426-431. [PubMed: 16163360]

Vernon R, Lara S, Drake C, Iruela-Arispe M, Angello J, Little C, Wight T, Sage E. Organized type I collagen influences endothelial patterns during "spontaneous angiogenesis in vitro": planar cultures as models of vascular development. In Vitro Cell Dev Biol Anim 1995;31(3):120-131. [PubMed: 7537585]

Weinstein B. What guides early embryonic blood vessel formation? Dev. Dynamics 1999;215:2-11.

Wu K, Gauthier D, Levine MD. Live cell image segmentation. IEEE T. Biomed. Eng 1995;42:1-11.

Wu X, Rabkin-Aikawa E, Guleserian KJ, Perry TE, Masuda Y, Sutherland FWH, Schoen FJ, Mayer JEJ, Bischoff J. Tissue-engineered microvessels on threedimensional biodegradable scaffolds using human endothelial progenitor cells. Am J Physiol Heart Circ Physiol 2004;287:H480-H487. [PubMed: 15277191]

Xu J, Tseng Y, Wirtz D. Strain hardening of actin filament networks. regulation by the dynamic crosslinking protein alpha-actinin. J Biol Chem 2000;275:35886-35892. [PubMed: 10954703]

Zamir EA, Czirók A, Cui C, Little CD, Rongish BJ. Mesodermal cell displacements during avian gastrulation are due to both individual cell-autonomous and convective tissue movements. Proc Natl Acad Sci U S A 2006;103:19806-19811. [PubMed: 17179040]

Zamir EA, Czirok A, Rongish BJ, Little CD. A digital image-based method for computational tissue fate mapping during early avian morphogenesis. Ann Biomed Eng 2005;33:854-865. [PubMed: 16078625]

Zammaretti P, Zisch AH. Adult 'endothelial progenitor cells' renewing vasculature. Int J Biochem Cell Biol 2005;37:493-503. [PubMed: 15618004] 



Figure 1.

Medial drift of the vasculature and the surrounding extracellular matrix. Endothelial cells are shown in a HH stage 6 quail embryo (Hamburger and Hamilton, 1951), labeled by microinjected, fluorophore-conjugated QH1 antibodies. The fibrillin-2 extracellular matrix (ECM) component was labeled by JB3 antibodies. Images capturing the fluorescence of both kind of dyes allow the characterization of the endothelial cell movements relative to the ECM surrounding. Panels A-J depict the early vascular plexus forming from disconnected endothelial cell clusters within a time course of 3 hours. The left panels (A, C, E, G and I) depict the vasculature (QH1 fluorescence, inverted for better visibility). The endothelium(red) and fibrillin-2-specific (green) fluorescence patterns are shown superimposed on the 
right panels (B, D, F, H and J). The blue arrowheads point to a vasculogenic sprout, which extends in a medial-caudal direction. Panel $\mathrm{K}$ depicts the fluorescence intensities along the dotted line in panel $\mathrm{J}$, collected from each time-lapse image within a 6 hour-long time interval. The topmost line of panel $\mathrm{K}$ is the pixel-array - constituting the marked line in panel $\mathbf{J}$ - from the first image of the sequence. Pixel-arrays from the consecutive image sequence are then placed under each other. Tilted lines thus represent the gradual medial motion of ECM (green) and vascular (red) tissue components. The two components usually generate lines with similar slopes, indicating co-movement. However, vasculogenic sprouts (marked by the blue arrowheads) move medially much faster then the surrounding ECM. 

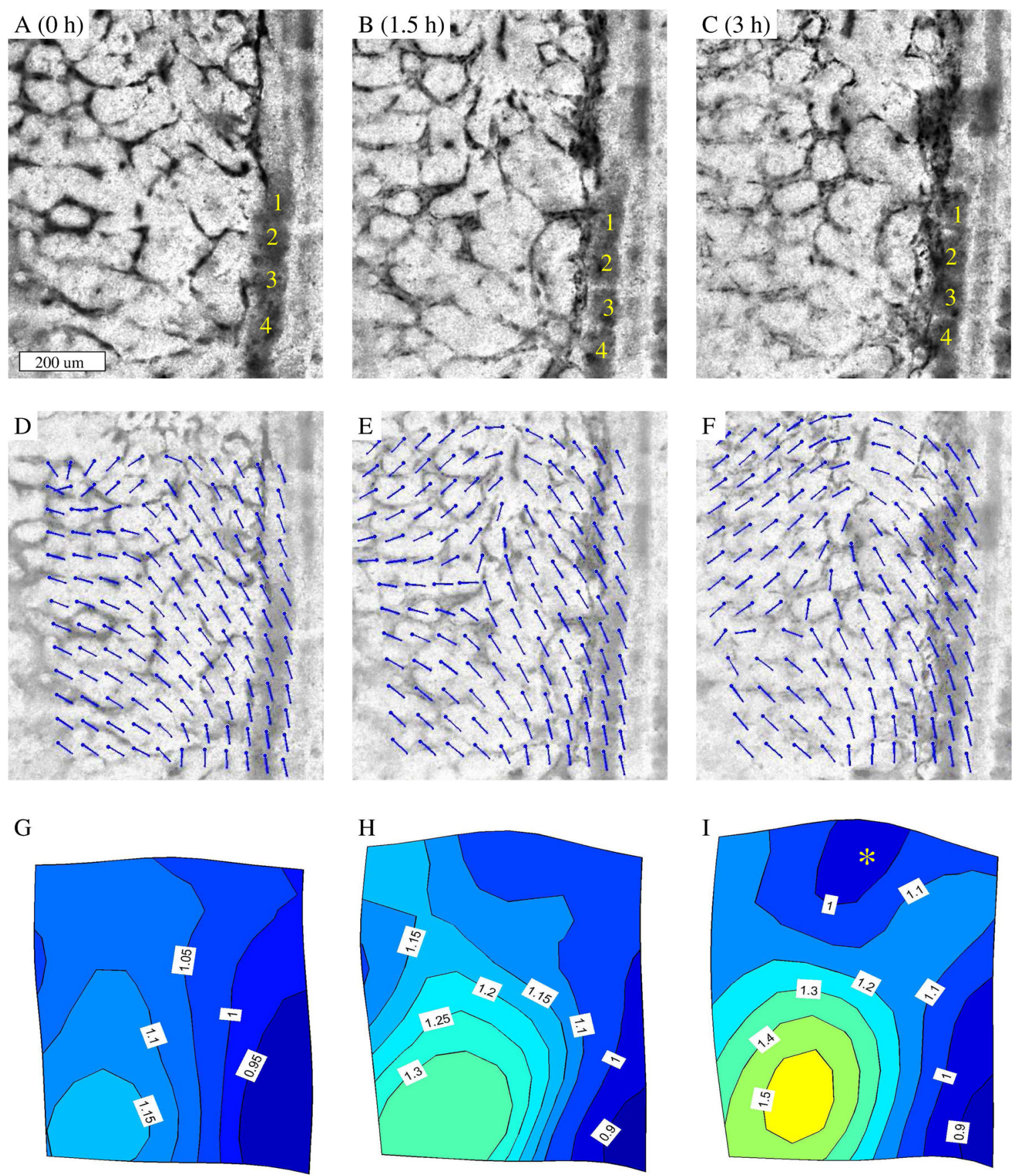

Figure 2.

Large-scale tissue movements contribute to the rearrangement of the nascent vascular plexus. Panels A-C depict changes in the QH1-labelled endothelium during a time course of 3 hours within a HH stage 7 quail embryo. The somites (marked from 1 to 4 ) appear as a non-specific background. The chosen time interval includes the formation of the dorsal aortae, major vessels assembling at both sides of the embryonic axis. Large vessels form by incorporating cells from adjacent smaller endothelial structures and by vascular fusion. The vasculature, together with the surrounding tissue, undergoes substantial deformations, calculated by particle image velocimetry (PIV) as described by Zamir et al. (2005). Panels D-F show the direction of the largest principal strain component, calculated from the tissue 
displacement PIV data. The area of the dorsal aortae is strained along the anterior-posterior axis. Tissue displacements also change the density (number of cells projected onto a unit area of the frontal plane) of the endothelial cells. Panels G-I show the relative change in the area of the traced tissue as a contour-line plot (delineated by the markers in the corresponding panels D-F). Numbers below 1 indicate density increase. The condensation at the area marked by the asterisk in panel I is the place of the forming sinus venosus - also characterized by extensive vascular fusion. 
Figure 3.

High resolution view of vasculogenic sprouting. Panels A-C depict changes in endothelial cell configuration during a time interval of 27 minutes. DIC images are shown superimposed with QH1 immuno-fluorescence (red). The extending cell protrusions eventually form a stable connection between two endothelial clusters, delineated with red in panel D. After Rupp et al. (2004). 


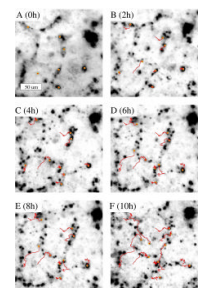

Figure 4.

Trajectories of individual primordial endothelial cells, relative to the surrounding vascular structures. The panels encompass a 10 hour long period of normal development. Yellow circles indicate the current position of representative QH1 positive cells; lines show their trajectory up to the corresponding time point. The embryonic midline is towards the right. After Rupp et al. (2004). 


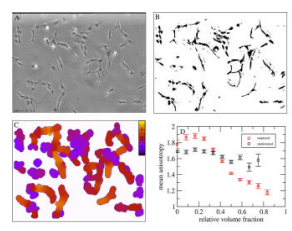

\section{Figure 5.}

Various cells, grown under standard culture conditions, can also exhibit "sprouts", i.e., long arrays of interconnected cells. Panel A depicts a phase contrast image of a C6 (rat glioma) cell line. To assay the non-random cell positions, the phase contrast image was segmented in a two-step procedure according to Wu et al. (1995). As a result, cell bodies appear as black clusters in panel B. The anisotropy of the local cell configuration (panel C) is characterized by a method employing a diffusion process where the diffusion coefficient is distributed according to a long-pass filtered version of panel $\mathrm{B}$. The obtained local anisotropy is dependent on the local cell density (panel D). Cell density is characterized by the relative volume fraction, the local volume fraction normalized by the maximal observed value (0.6 for C6 cells). Thus, the unit of relative volume fraction corresponds to confluent cells. As C6 cells are themselves elongated, we compared the obtained data to simulated configurations where each cell body is randomly placed on the plane. While the cell bodies of C6 cells are anisotropic themselves, the configuration of adjacent cells significantly increase the anisotropy if the local cell density is sufficiently sparse (below $30 \%$ confluency). 


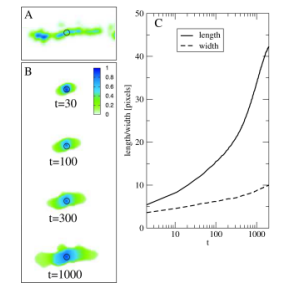

\section{Figure 6.}

Calculation of anisotropy index. A diffusive process is started from a selected point of the image (circle). The diffusion coefficient changes between 0 and 1 according to the pixel values in the segmented and smoothened image (panel A). The selected point acts as a pointsource of the diffusive field, where the concentration is kept steady. During the simulated diffusion process a gradually increasing area emerges around the point source (panel B). The growing area is characterized by its length and width (panel C), calculated from the principal moments of the concentration field depicted in panel B. The local anisotropy is characterized as a ratio between the area length and width at the time point when the width reaches the typical syze of a single cell. 
A
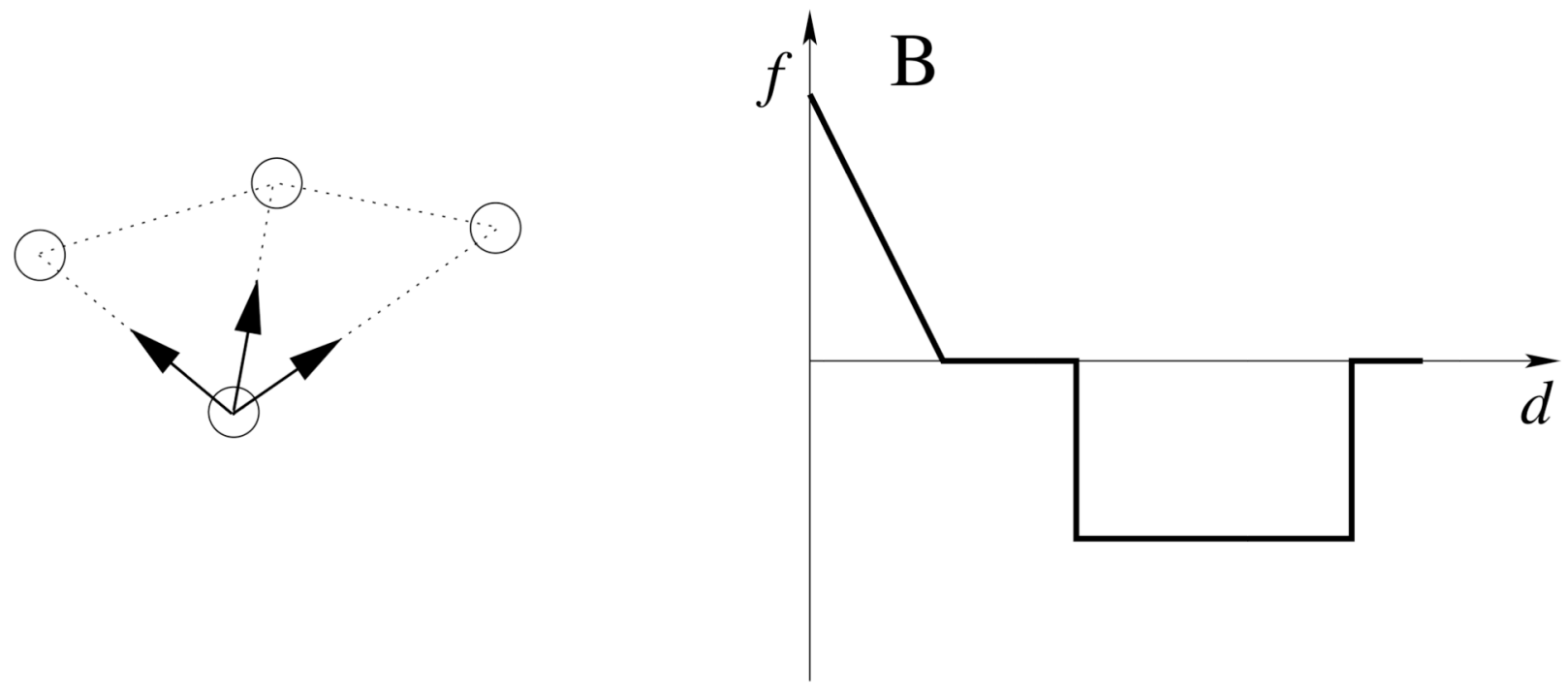

Figure 7.

Schematic representation of the model. Panel A depicts particles (circles) interacting with their neighbors (dashed lines). The interaction results in deterministic drift motion (arrows) along the line connecting the particles. The direction and magnitude $f$ of the drift is determined by the distance $d$ between the particles. If particles are close, the repel each other $(f>0)$. If the distance is within a pre-determined range, they tend to move closer $(f<0)$. The strength of the attraction is modulated by the anisotropy of the environment (not depicted). 


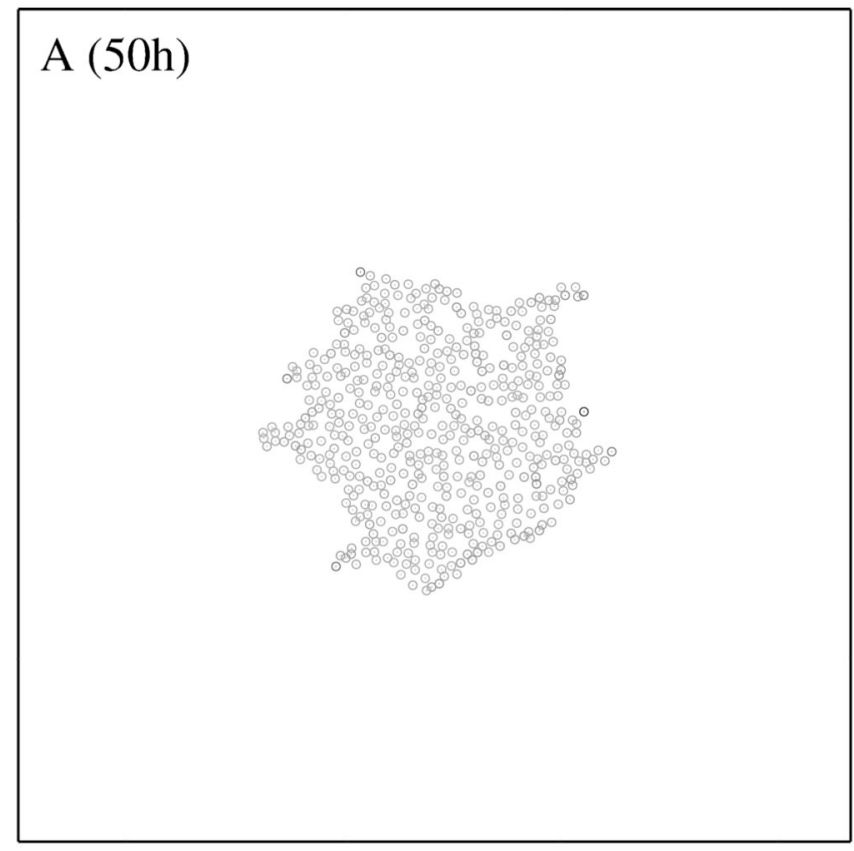

B (150h)

\section{C (350h)}

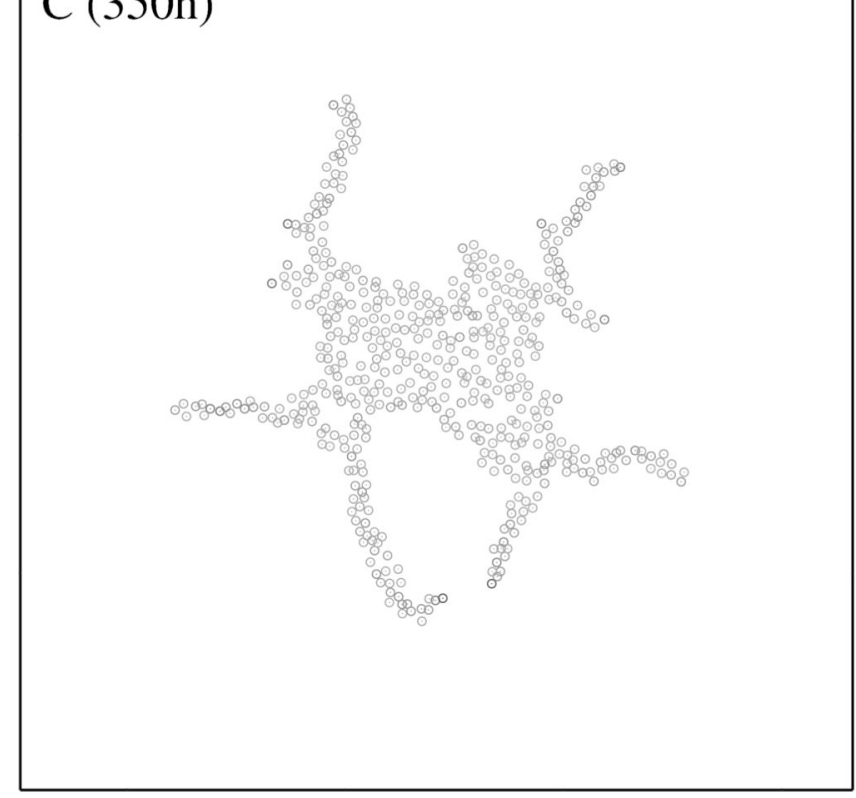

\section{$\mathrm{D}(500 \mathrm{~h})$}
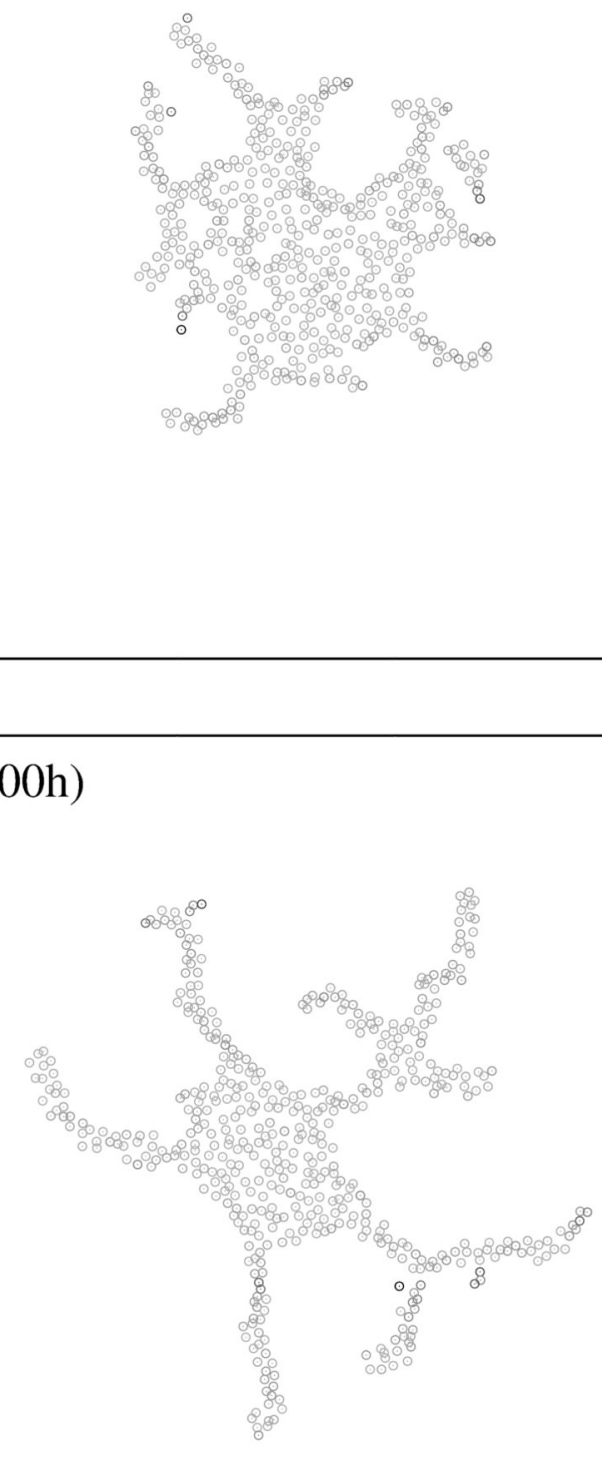

Figure 8.

Time development of the model consisting of particles preferentially attracted to anisotropic structures. When $N=500$ particle are started from a dense cluster (A), branches form (B) which slowly elongate $(\mathrm{C}$ and $\mathrm{D})$. The gray level of the particles indicate the anisotropy of their local environment. The linear size of the area shown is $L=40 R \approx 1.6 \mathrm{~mm}$. 

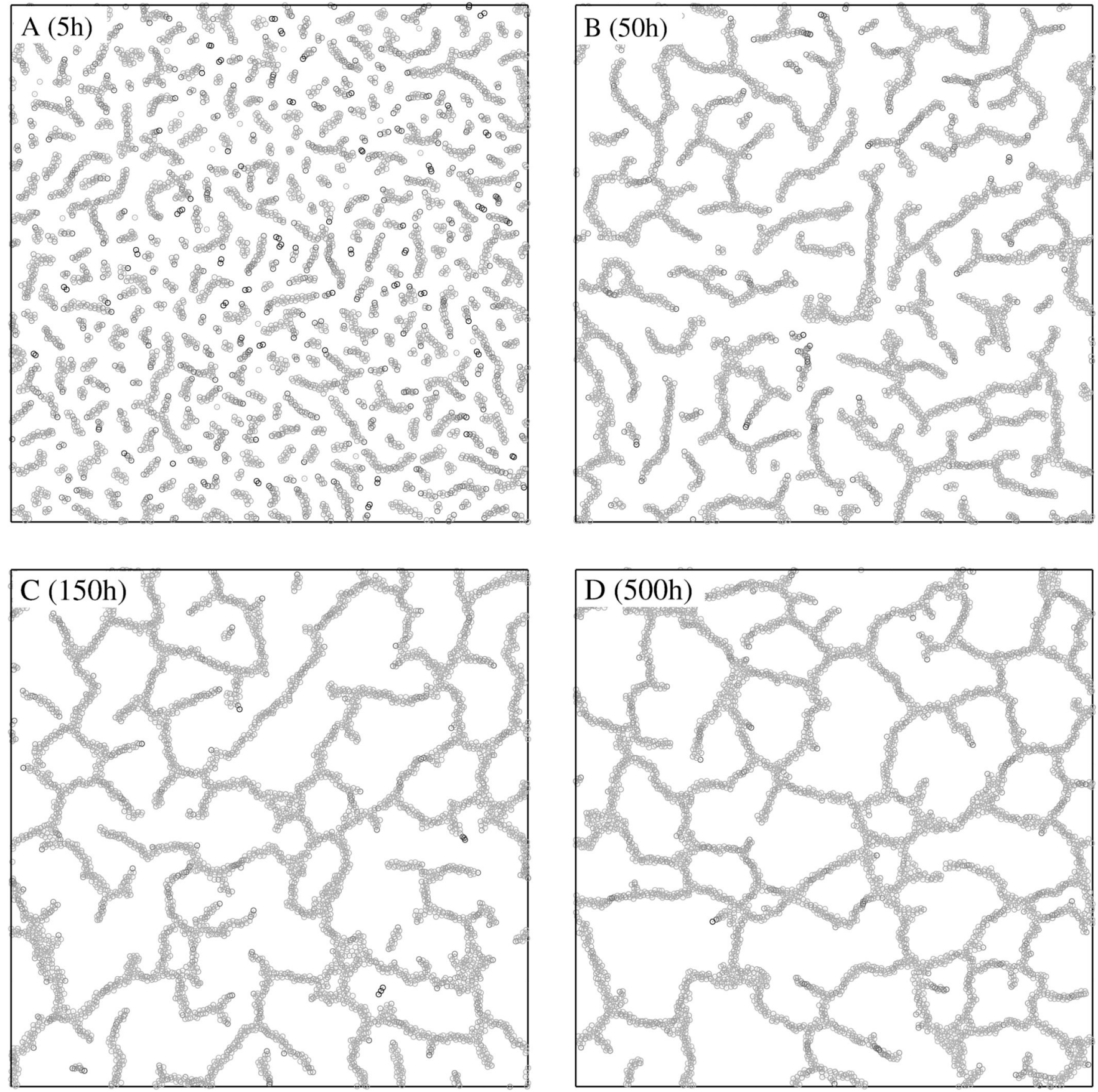

Figure 9.

Time development of the model consisting of particles preferentially attracted to anisotropic structures, started from a random initial configuration. $N=5000$ particles assemble in growing linear segments (panels A and B). An interconnected network develops if the number of particles is sufficiently large (C). After an initial transient period a quasistationary state emerges where the formation of new branches offset the occasional damage of random particle motion, and the characteristic size of avascular areas do not change (compare panels $\mathrm{C}$ and $\mathrm{D}$ ). The linear size of the area shown is $L=80 R \approx 3.2 \mathrm{~mm}$. 

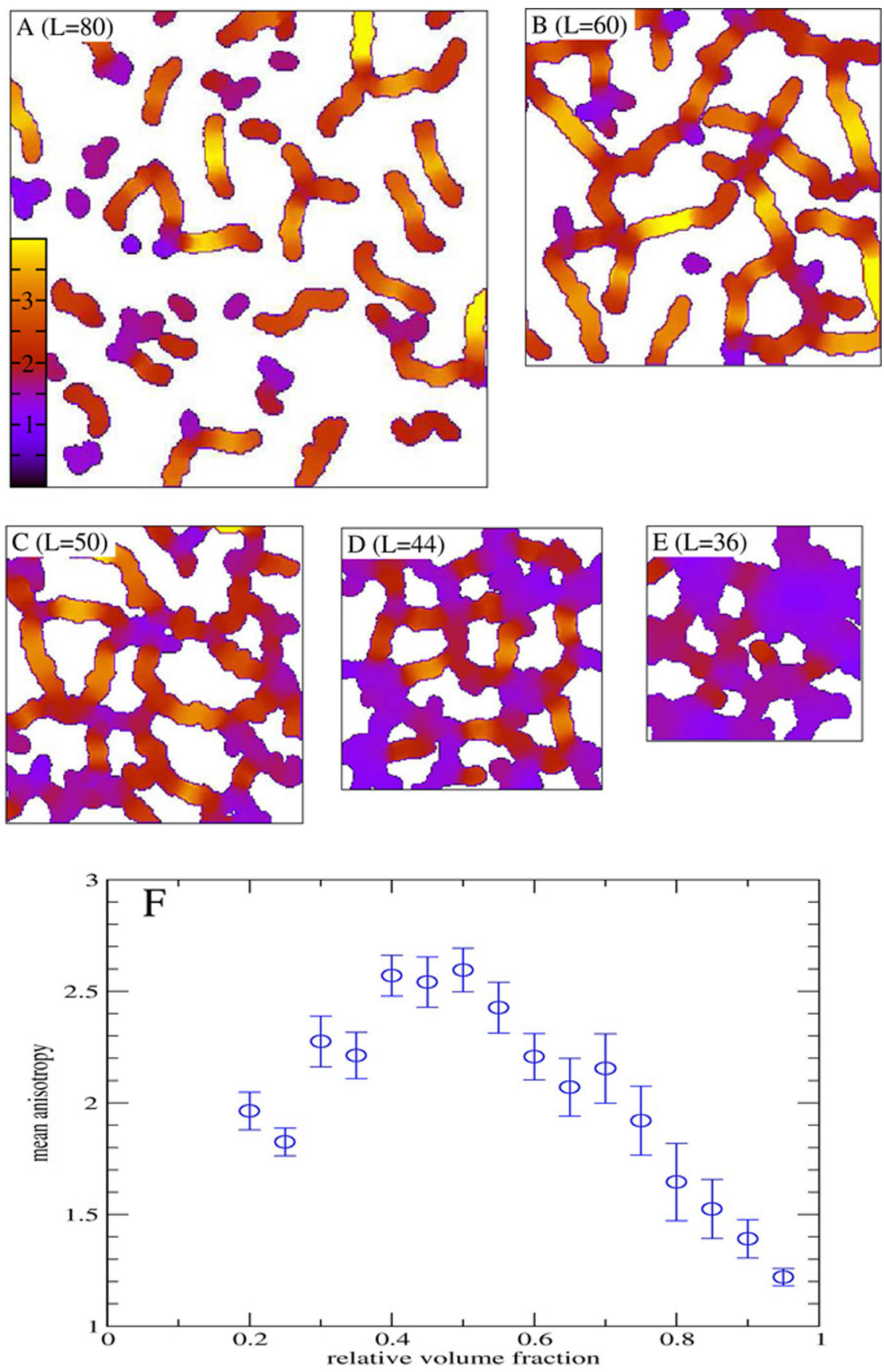

Figure 10.

Depending on particle density, the model yields long elongated clusters or an interconnected network. $N=2000$ particles are placed in a square area of size $L=80 R(\mathrm{~A}), 60 R(\mathrm{~B}), 50 R$ $(\mathrm{C}), 44 R(\mathrm{D})$ and $36 R(\mathrm{E})$, with periodic boundary conditions. The calculated local anisotropy map is superimposed on the configurations obtained at the quasi-stationary state of the simulations. Such simulations allow the determination of the average anisotropy of the configurations as a function of local particle density (F), to be compared with panel D of Fig. 5. 